\title{
TMS Intensity and Focality Correlation with Coil Orientation at Three Non-Motor Regions
}

This paper was downloaded from TechRxiv (https://www.techrxiv.org).

\section{LICENSE}

CC BY 4.0

\section{SUBMISSION DATE / POSTED DATE}

20-07-2021 / 04-08-2021

\section{CITATION}

Gomez-Feria, Jose; Fernandez-Corazza, Mariano; Martin Rodriguez, Juan Francisco; Mir, Pablo (2021): TMS Intensity and Focality Correlation with Coil Orientation at Three Non-Motor Regions. TechRxiv. Preprint. https://www.techrxiv.org/articles/preprint/TMS_Intensity_and_Focality_Correlation_with_Coil_Orientation_at_Th. Motor_Regions/15023409

DOI 


\title{
TMS Intensity and Focality Correlation with Coil Orientation at Three Non-Motor Regions
}

\author{
Jose Gomez-Feria, Mariano Fernandez-Corazza, Juan F Martin-Rodriguez, Pablo Mir
}

\begin{abstract}
Objective: The aim of this study is to define the best transcranial magnetic stimulation (TMS) coil orientations for three clinically relevant brain areas (presupplementary motor area, inferior frontal gyrus, and posterior parietal cortex) by means of simulations in 12 realistic head models of current density. Methods: We computed the current densities generated by TMS in our three volumes of interest (VOI) that were delineated based on published atlases. We then analyzed the maximum intensity and spatial focality for the normal and absolute components of the current density considering different percentile thresholds. Lastly, we correlated these results with the different anatomical properties of our VOls. Results: Overall, the spatial focality of the current density for the three VOls varied depending on the coil's orientation. There was major interindividual variability, and there was therefore no specific orientation that produced the best focality for all subjects. Further analysis showed that the differences in individual brain anatomy were related to the amount of focality achieved. In general, a larger percentage of sulci resulted in larger spatial focality. Moreover, larger normal current density intensity was achieved when positioning the coil axis perpendicular to the predominant orientations of the gyri of each 's VOI. Conclusion: For a rough approximation, better coil orientations can be based on the individual's specific brain morphology at the VOI. Moreover, TMS computational models should be employed to obtain better coil orientations in non-motor regions of interest. Significance: Finding better coil orientations in non-motor regions is a challenge in TMS and seeks to reduce interindividual variability. Our individualized TMS simulation pipeline leads to lesser interindividual variability in the focality, likely enhancing the efficacy of the stimulation and reducing the risk of stimulating adjacent, nontargeted areas.
\end{abstract}

Index Terms- Brain stimulation, coil orientation, computer simulation, finite element analysis, patientspecific modeling, transcranial magnetic stimulation (TMS).

This work was supported by the Asociación Universitaria Iberoamericana de Postgrado (AUIP), the Instituto de Salud Carlos III-Fondo Europeo de Desarrollo Regional (PI14/01823, PI16/01575, PI18/01898, PI19/01576), Consejería de Economía, Innovación, Ciencia y Empleo de la Junta de Andalucía (CVI-02526, CTS-7685), Consejería de Salud y Bienestar Social de la Junta de Andalucía (PI-0471-2013, PE0210-2018, PI-0459-2018, PE-0186-2019), the Spanish Ministry of Science and Innovation "Retos Colaboración" 2019 program (TC2019-007150-1), the ANPCyT PICT2017-0857 and UNLP I-209 grants. J. Gomez-Feria was supported by the Ministerio de Ciencia e Innovación (Grant FPU16/03550) and Juan F Martin-Rodriguez was supported by the VI-PPIT-US from the University of Seville (USE-18817-A). (J. GomezFeria and M. Fernandez-Corazza contributed equally to this work). (Corresponding authors: Juan F Martin-Rodriguez and Pablo Mir).

J. Gomez-Feria, J. F Martin-Rodriguez and P. Mir are with the Unidad de Trastornos del Movimiento, Servicio de Neurología y Neurofisiología Clínica, Instituto de Biomedicina de Sevilla, Hospital Universitario Virgen del Rocío/CSIC/University of Seville, Seville, Spain, and the Centro de Investigación Biomédica sobre Enfermedades Neurodegenerativas (CIBERNED), Madrid, Spain. (e-mail: jf.martin.rodriguez@gmail.com and pmir@us.es).

M. Fernandez-Corazza is with the LEICI-Instituto de Investigaciones en Electrónica, Control y Procesamiento de Señales, Universidad Nacional de La Plata (UNLP), CONICET, La Plata, Argentina.

\section{INTRODUCTION}

Transcranial magnetic stimulation (TMS) is a non-invasive brain stimulation technique based on Faraday's electromagnetic induction principle [1]. Electric current is applied to a circular coil placed on the subject's scalp, generating magnetic fields that penetrate the brain, with significant attenuation of the signal due to varying tissue conductivity and depth. These magnetic fields induce an current density (Efield) in the neural tissue, depolarizing the cells and increasing cortical excitability [2]. The resultant current density depends highly on the type of coil and its position on the scalp, with the figure- 8 coil the most standardized model for achieving the largest focality [3]. The type of pulse generated can be monophasic (i.e., single or paired pulse) or biphasic (i.e., repetitive TMS), depending on the aim of the TMS, which can be used for modulating brain functions and has important clinical applications [4-8]. Depending on the purpose of the stimulation, there is interest in finding the coil orientation that induces the largest current density intensity within a volume of interest (VOI) (i.e., in single-pulse TMS) or reduces the stimulation in other brain regions beyond the VOI (i.e., in repetitive TMS) leading to possible side effects or unwanted plastic changes. For the first situation (single or paired pulse), it is important to analyze the perpendicular component of the induced current density [9-11]. For the second scenario (repetitive stimulation), it is important to study the spatial focality of the induced current density [12], defining spatial focality as the ratio of the current density in the VOI to the current density in the total grey matter (GM) (above a certain threshold).

Numerous studies have emphasized the idea that the effectiveness of transcranial magnetic stimulation depends highly on the coil orientation relative to the subject's head [10, 11, 13-16], which implies that the direction of the induced current density has a major effect on the efficacy of TMS, an issue that has been widely investigated in the motor cortex (M1) $[7,10,11,13,14,17-21]$. These studies have shown that the 
best coil orientation for M1 is obtained when the coil is placed 45 degrees to the medial-sagittal plane of the subject's head, a finding corroborated by computational simulations of TMS in $\mathrm{M} 1[22,23]$. This orientation induces a posterior-anterior (P-A) current density that is perpendicular to the central sulcus. This coil orientation is the best one for most individuals due to the similarity in anatomical morphology and orientation of the motor cortex and central sulcus [24]. However, these interindividual anatomical similarities are not usually found in other brain regions, such as non-motor regions, where sulci and gyri distributions tend to vary $[12,24,25]$. Thus, differences in morphological features within the brain's anatomy play an essential role in determining the coil orientation that induces the largest spatial focality of the current density in the VOI [25, 26].

Various theories have been proposed to explain why the perpendicular component of the current density plays such an important role in generating an optimal cortical response in M1, given that the direction of the normal component of the electric field along the pyramidal apical dendrites likely influence neuronal activation $[9,10,18,27-29]$. However, the most accepted explanation is provided by the theoretical cortical column cosine model of TMS efficacy (C3-model) [9], in which the cortical column behaves as a functional unit where the vector of the current density induced by TMS is decomposed into two components: 1) the perpendicular component of the current density and 2) the component parallel to the cortical column, which is the product of the absolute value of the current density and the cosine angle between the column and the current density orientations. The authors of the $\mathrm{C} 3$-model state that the electric field perpendicular to the cortical surface contributes the most to the resultant TMS-induced brain activation. VOIs within the brain with larger numbers of sulci would therefore include regions with more cortical surfaces perpendicular to the TMS stimulation, thereby inducing stronger brain activation. This model also indicates that the current density decreases with depth, inducing current densities below a certain activation threshold, thereby making the signal too weak to generate a cortical response. The importance of current density depth penetration is also mentioned in the study by Sorkhabi et al. [30], which emphasized the need for determining focal and depth current densities for the correct stimulation of specific brain regions and concluded that weak current densities do not generate a sufficiently high response to properly stimulate specific neuronal populations [3].

TMS studies in non-motor brain areas have found differing rules regarding coil orientations but have been unable to find an optimal coil orientation for all individuals such as those identified for M1. This lack of optimal coil orientation in cortical areas outside M1 can be due to a high inter-subject variability in anatomical features [10, 31-36]. Janssen et al. studied the optimal coil orientations for a wide range of nonmotor cortical regions but using only one head model [10]. The authors found the coil orientation that would induce the largest effective current density in these regions without analyzing the field's focality. These simulations were performed on a general but highly realistic head model, and the selected target regions were spheres measuring $3 \mathrm{~mm}$ in radius, with their centers located on the cortical surface. However, this VOI selection method might be inaccurate because a sphere does not represent the actual volume that contains the region of interest of a specific individual. Although this work concluded that there are better coil orientations for different non-motor regions, the results might be inconclusive because the authors only used one head model, and the potential interindividual variations in brain anatomy could potentially vary the optimal coil orientations. The authors also did not use an intensity threshold to select only those current densities that could cause a neural effect [37]. Several studies have indicated a threshold of approximately 50 $\mathrm{V} / \mathrm{m}$ for an electric field capable of stimulating neuronal populations in TMS [30,38]. As in these studies, we focused on analyzing the spatial focality of TMS over the various VOIs that would minimize the potential undesirable effects of repetitive TMS.

The purpose of our study was to analyze, in 12 subjects, whether there is a better coil orientation in terms of spatial focality for the treatment of three non-motor VOIs that play an important role in various conditions: the pre-supplementary motor area (pre-SMA), which is likely related to Parkinson's disease, Tourette syndrome, and central fatigue [39-41]; the inferior frontal gyrus (IFG), which is involved in Tourette syndrome [42, 43]; and the posterior parietal cortex (PPC), which is correlated with dystonia [44]. For consistency, we defined each of these VOIs in a common atlas space, applying posteriorly a matrix transformation to each subject's native space. We also analyzed the importance of the perpendicular component of the current density to achieve the largest average field within the VOI and its correlation with the position of the coil's handle, although these stronger intensity coil positions might lead to lower spatial focality. An important goal of this study is to correlate the best coil orientation established by spatial focality with the individual geometrical characteristics of the VOIs. We hypothesize that, due to interindividual changes in anatomical brain properties, there is no globally preferred coil orientation for maximizing the spatial focality, but there are optimal orientations for each individual. These improved orientations might be related to the distribution of sulci and gyri within each individual's VOI. We also hypothesize that the largest effective current density (the average of the normal-to-cortex component of the current density) is obtained when the coil's handle is mostly oriented perpendicular to the sulci walls of the VOI.

\section{METHODS}

Subjects

Twelve healthy individuals (5 women, 7 men, mean age 30 \pm 4 years) participated in the study. Magnetic resonance images (MRI) were taken at the University Hospital Virgen del Rocío (Seville, Spain) and inspected by doctors and technicians to corroborate the absence of any possible conditions and ensuring that the selected subjects were healthy. The subjects were all informed about the MRI procedure and signed the informed consent. The study was approved by the ethics committee of University Hospital Virgen del Rocío and followed the standards of the Declaration of Helsinki.

\section{Imaging}

We acquired structural $\mathrm{T} 1$ and T2-weighted MRI scans using a 3T MRI scanner (Ingenia, Philips Healthcare) and a 16channel SENSE head. T1-weighted scans were acquired using 
the following parameters: repetition time (TR)/echo time (TE), 8.2/3.8 ms; flip angle 8; field of view (FOV) $240 \mathrm{~mm}$; section thickness $1 \mathrm{~mm}$; matrix $240 \times 222$; and voxel size $0.94 \mathrm{~mm}$. The T2-weighted scans were acquired using the following parameters: TR/TE 3000/80 ms; FOV $245 \mathrm{~mm}$; section thickness $4 \mathrm{~mm}$; and matrix $444 \times 315$.

\section{Geometrically realistic head models}

Before calculating the current densities induced by TMS in the various subjects, the first step was the construction of individual and highly realistic tetrahedral head models. The T1 and T2-weighted structural MRI images were segmented and meshed using the SimNIBS pipeline $[45,46]$. The meshes contained eight different tissues with five different conductivities (Supplementary Table S1) [19, 20], namely white matter, GM, cerebrospinal fluid, skull, eyes, sinus cavities, air cavities and scalp (Supplementary Fig. S1). Within SimNIBS, we used the command 'headreco' to create the model, which relied on SPM12 and CAT12 for segmentation [45]. The advantage of using SimNIBS is that it is a comprehensive and fully automated process that requires no manual intervention. We used SimNIBS for the geometrical head model construction but not for the coil placement or the electromagnetic simulations, for which we used custom MATLAB scripts

\section{Definition of target region}

Brain regions on the right hemisphere were selected based on published atlases, selecting the pre-SMA from Sallet's dorsal frontal connectivity-based parcellation atlas [47], IFG from the Harvard-Oxford cortical structural atlas [48], and PPC from the Talairach atlas $[49,50]$. Using the command flirt FSL linear transformation tool [51-53] and the standard2example matrix, we registered each subject's brain to the Montreal Neurosciences Institute (MNI) 152 space and extracted the selected VOIs based on the corresponding atlas. We then performed a reverse normalization using an inverse matrix transformation to convert these regions back to each subject's native space. Following this procedure, we were able to minimize the interindividual errors in the VOI selection (Fig. $1)$.

\section{Induced E-field modeling}

The electrical forward problem in TMS can be stated as follows: given the electrical head model, a coil model and location, the electrical conductivities of the various tissues and a stimulator intensity, calculate the electrical field in the head, more precisely in the GM volume. This problem is governed by Maxwell's equations, where the resulting electric field $\mathrm{E} \rightarrow$ in the conductor volume can be defined as follows [54]:

$$
\vec{E}=-\nabla \phi-\frac{\partial \vec{A}}{\partial t}
$$

where $\phi$ is the electric potential and $\mathrm{A}$ is the magnetic vector potential. The second term on the right side of Equation $1((\partial \mathrm{A}$ $\vec{J} / \partial \mathrm{t})$ is the primary field, which depends only on the coil location and can be calculated analytically. The first term $(-\nabla \phi)$ is the secondary electric field that is produced by the induced currents and depends on the volume geometry and conductivities. This secondary field requires solving the
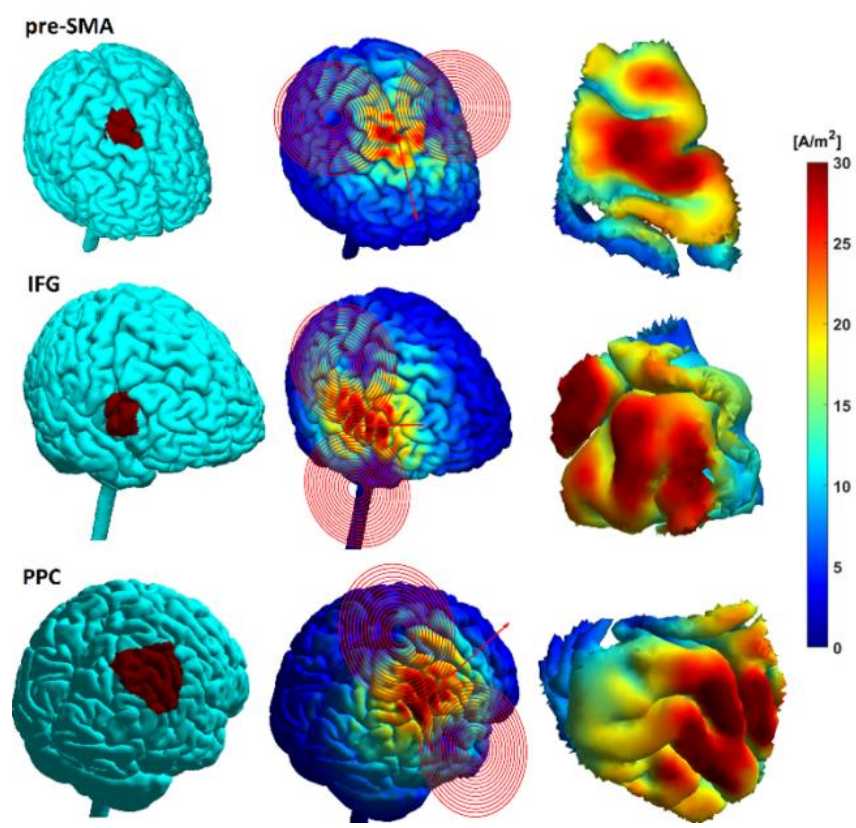

Fig. 1. VOI selection: pre-SMA, IFG, PPC. The left column shows the VOIs painted in red. The second column shows an example of the TMS induced electric filed at $0 \%$ threshold percentile. The right figure shows only the current density module at the VOI.

following Poisson equation [54] secondary electric field that is produced by the induced currents and that depends on the volume geometry and conductivities. This second field requires solving a Poisson equation [54]:

$$
\nabla \cdot(\sigma \nabla \phi)=-\nabla \cdot\left(\sigma \frac{\partial \vec{A}}{\partial t}\right)
$$

where $\sigma$ represents the electrical conductivity tensor with the Neumann boundary condition that the electric current does not leave the volume (i.e., $(\sigma \nabla \phi) \cdot \mathrm{n} \overrightarrow{\mathrm{n}}=0$, where $\mathrm{n} \overrightarrow{\text { is }}$ the normal vector of the boundary). The left side of Equation $2(\nabla \cdot(\sigma \nabla \phi))$ is equivalent to the Poisson equations that govern the forward problems of electroencephalography, transcranial electrical stimulation and electrical impedance tomography [55], [56]. The right side of Equation 2 is the difference, i.e., the source term. In Equation 2, the sources of the scalar potential $\phi$ are the components of the primary electric field.

We modelled two 8-shaped commercial coils: the Magstim D70 Alpha flat coil (70 $\mathrm{mm}$ in diameter) (Magstim, Wales, UK) and the Magstim D40 Alpha flat coil (40 $\mathrm{mm}$ in diameter). The characteristics of these coils, such as number of turns and the internal/external radius, were required to run the simulations and were extracted from other studies [11, 57, 58]. The characteristics we employed to model the D70 coil where as follows: distance between centers, $47 \mathrm{~mm}$; minimum radius, 28 $\mathrm{mm}$; maximum radius, $45 \mathrm{~mm}$; and 9 turns. For the D40 coil, the characteristics where as follows: distance between centers, $38 \mathrm{~mm}$; minimum radius, $6 \mathrm{~mm}$; maximum radius, $36 \mathrm{~mm}$; and 15 turns. Both coil models $(40 \mathrm{~mm}$ and $70 \mathrm{~mm}$ ) presented the coil's handle tangential and not perpendicular to the coil. We placed each coil in the plane tangential to the scalp, with the coil center placed at the closest point of the scalp to the center of the VOI and separated from the scalp by $5 \mathrm{~mm}$, following the study by Laakso et al. [11]. Supplementary Fig. S2 shows the models of the two coils employed $(40 \mathrm{~mm}$ and $70 \mathrm{~mm}$ ). 
We solved the primary field distribution in Equation 1 analytically in terms of elliptical integrals, following the study by Wang et al. [54] in equation 34, assuming a source current derivative in the coil of $165 \mathrm{~A} / \mu \mathrm{s}$, as described in Laakso et al. study [11] for the Magstim 2002 ${ }^{2}$ stimulator. The electric potential $\phi$ was calculated from Equation 2 numerically using our in-house first-order finite element method with the Galerkin approach [59, 60]. We solved the forward problem for eight coil orientations ( $45^{\circ}$ steps) (Fig. 2) and the two types of coils, for each subject and for each VOI. Given the lack of substantial differences in the results related to the orientations of the two types of coils, we focused the analysis only on the $40-\mathrm{mm}$ coil simulations. We opted for this coil instead of the 70-mm coil because the evaluated regions are more difficult to target (and the 40-mm coil is expected to be more focal) and because the $40-\mathrm{mm}$ coil is the one used in our laboratory.

\section{Metrics of evaluation}

To obtain the best simulation angle, we studied the spatial focality of the module, normal, and tangential components of the current density (Supplementary Fig. S3) to determine the accuracy of the spatial targeting and the avoidance of stimulating non-target brain regions, which could result in negative clinical side effects [3]. We defined the spatial focality as the ratio of the integral of the current density in the VOI and above a certain threshold to the integral of the current density induced in the whole GM (also above the same threshold). This definition was applied to a set of thresholds of the current density determined as percentiles of the total current density: $0 \%, 10 \%, 50 \%$, and $65 \%$ (Fig. 3). For the analysis, we focused on the results for the $65 \%$ percentile, which was a conservative threshold for most of the subjects and orientations, considering the $50 \mathrm{~V} / \mathrm{m}$ neuronal activation threshold $[30,38]$.
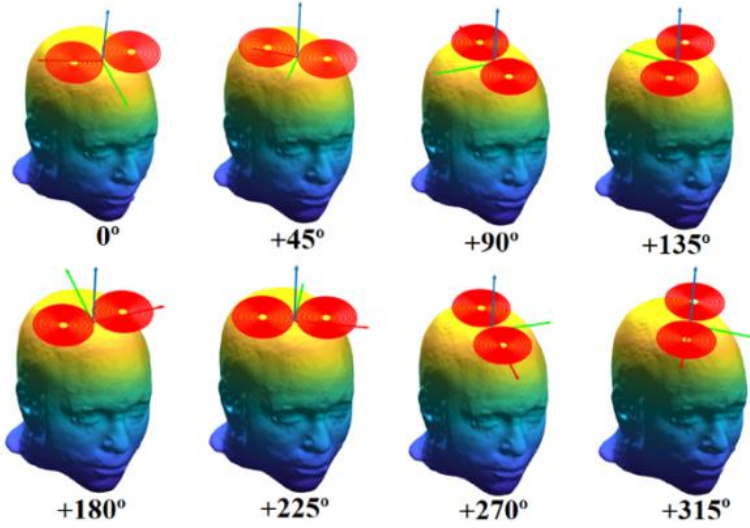

Fig. 2. Coil rotation. Example of the different coil rotations over preSMA every $45^{\circ}$ and using the $40 \mathrm{~mm}$ coil. The same rotation angles were applied over IFG and PPC to find the best coil orientations. The green vector represents the direction of the coil handle

\section{Anatomical analysis}

Lastly, we studied the relationship between the coil orientations and the anatomical properties of our VOIs by analyzing the sulci and gyri geometry within each VOI. We separated the wrinkled cortical surface within each VOI into two portions: surface mostly parallel to the coil plane (mostly gyri) and surface mostly perpendicular to the coil plane (mostly sulci). This separation was done by computing the angle between the normal vector to the coil plane and setting a
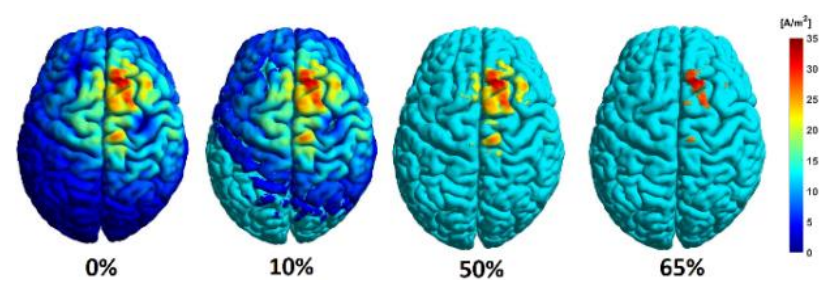

Fig. 3. Working with threshold percentiles. Different threshold percentiles were applied in each stimulation. $0 \%$ shows the full stimulation without any cutoff value. $65 \%$ only draws intensities over that percentile. Only intensities over that threshold are expected to generate direct cortical response.

threshold of 45 degrees to separate these two surface components. We first calculated the percentage of sulci and gyri within a selected region (i.e., pre-SMA, IFG, or PPC) and compared this percentage with the spatial focality in our 12 subjects, obtaining a linear regression that correlated the spatial focality and sulcus or gyrus percentage. Next, we calculated the average direction of the normal vector of the sulcus within the VOI and compared it with the direction of the predominant component of the TMS induced current density (which, in a tangential figure-eight coil, equals the direction of the coil's handle). With this analysis and knowing that the perpendicularto-the-cortex component of the current density plays an important role in obtaining the best cortical response, our goal was to find a method for predicting the best coil orientation by knowing only the direction of the normal sulcus' vector. We further assessed the association between the current density intensity and the alignment of normal vector sulci with the normal component of the current density with linear mixed effect models in the three VOIs. As fixed effects, we entered the alignment of normal vector sulci with the normal component of the current density, and the subject was entered as a random effect.

\section{RESULTS}

The mean spatial focality values for the three VOIs and eight coil orientations are listed in Figure 4. There was not a same coil orientation that produced the largest focality for all subjects. However, all coil orientations showed an overall similar spatial focality close to $60 \%$ for pre-SMA and IFG, and close to $88 \%$ for PPC. The polar plots in Figure 4 show the differences in spatial focality between the individual subjects for each coil orientation. The bottom images of Figure 4 represent an example of a random subject with two coil orientations at the maximum and minimum spatial focality.

A two-way ANOVA introducing VOI and coil orientation as intraindividual factors revealed a significant main effect for VOI $(\mathrm{p}<0.0001)$ but not for coil orientation or the interaction. Higher focality values were obtained for PPC, regardless of the coil orientation (Tukey's honestly significant difference, $\mathrm{p}<0.0001$ ) (Fig. 5). We also calculated the average value of the sulci proportion of the 12 subjects for the different VOIs, demonstrating a significant difference between the percentage of sulci in PPC when compared with the pre-SMA and IFG (Fig. 


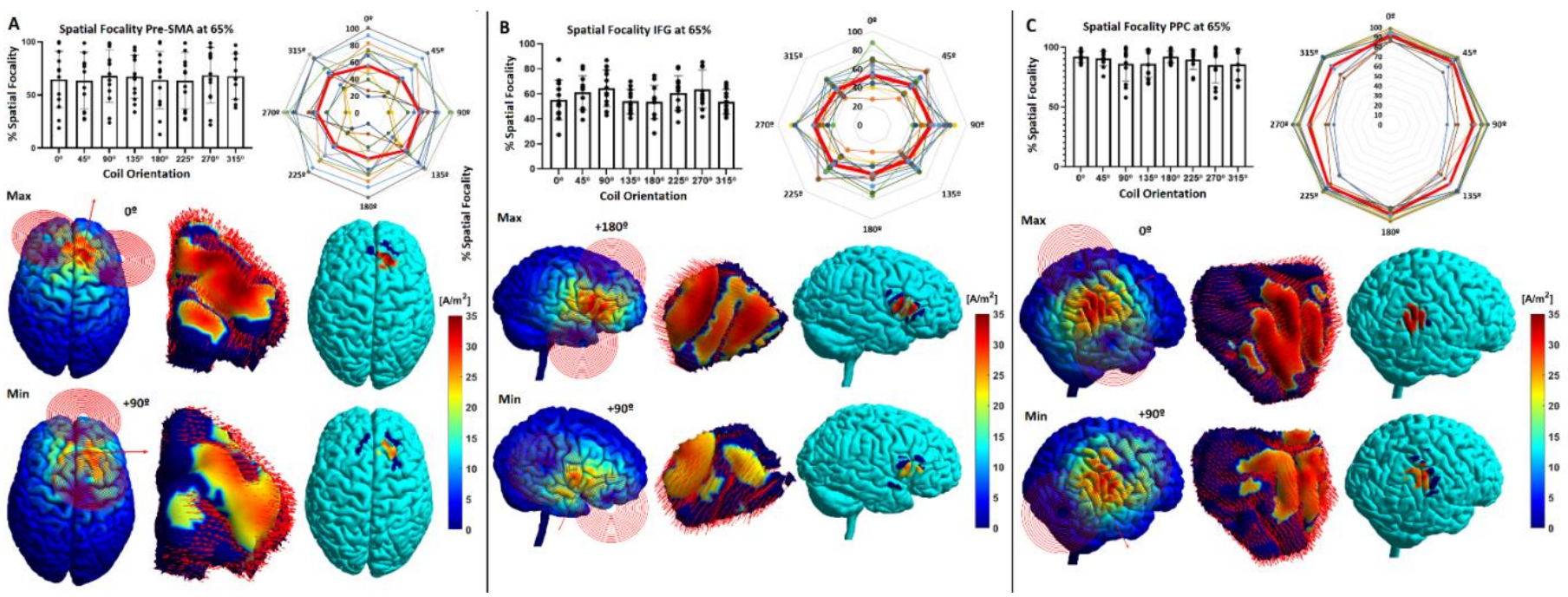

Fig. 4. Spatial Focality at pre-SMA (A), IFG (B) and PPC (C). The bar plots represent the average value of spatial focality at percentile $65 \%$ for the 12 subjects, demonstrating no significant differences between different coil orientations. Each dot represents the value of spatial focality of an individual subject for a selected coil orientation, showing a high variability between subjects when fixing the coil at one position. The polar plots represent the spatial focality values for the 12 subjects with different colors per subject. Red gross line of the polar plots shows the average value for each coil orientation for all subjects. Looking at the bar plots it can be seen a slight increase of spatial focality in $90^{\circ}$ and $270^{\circ}$ for IFG, and $0^{\circ}$ and $180^{\circ}$ for PPC. However, these differences were not significant. Bottom images represent examples of the current density in $\mathrm{A} / \mathrm{m} 2$ induced by TMS above the $65 \%$ percentile. The figures on the right show this stimulation, painting in dark blue the stimulated volumes outside the VOI. For each region, one representative subject was selected to represent maximum (top row) and minimum (bottom row) spatial focality depending on coil orientation.

\section{VOls Comparison}

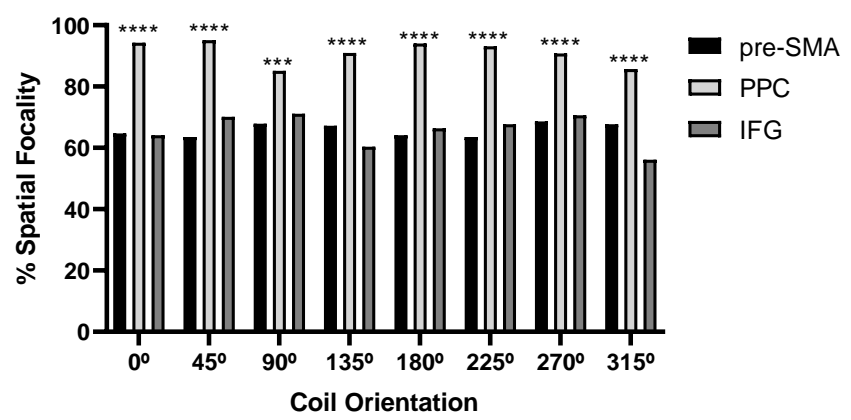

Fig. 5. Multiple comparison between pre-SMA, PPC and IFG for each coil orientation. Using a two-way ANOVA and Tukey's multiple comparison test, differences of spatial focality between regions were analyzed. PPC presented a significant difference $(\mathrm{p}<0.0001)$ with pre-SMA and IFG, showing on average $88 \%$ of spatial focality, compared with the $60 \%$ of pre-SMA and IFG.

6). In TMS, the current moves parallel to the coil plane, and the presence of more sulci walls in the VOI that are perpendicular to this plane implies that there is a larger area in the VOI for the electric current to penetrate these walls and enter the brain perpendicularly, thereby increasing the VOI intensity to the outside-VOI intensity ratio (i.e., the spatial focality), which might explain why the PPC in Figure 5 achieved the largest spatial focality on average. Further analysis revealed a significant linear relationship between the overall percentage of sulci in the VOI and the spatial focality achieved in the preSMA and IFG but not in the PPC (Fig. 7). Conversely, negative relationships were found between the percentage of gyri and spatial focality in the three VOIs.

Next, we analyzed whether the alignment of the coil's orientation with the normal vectors of the sulci could predict the intensities of the normal component of the current density induced by TMS within the three VOIs. Results from the linear mixed-effects models showed that a better alignment of the coil handle with most of the normal vectors of the sulci surface was significantly associated with larger intensity of the normal component of the current density, which held true for the three VOIs. These analyses were performed for all coil orientations and for all subjects (Fig. 8). We found no significant correlations between the spatial focality and this alignment.

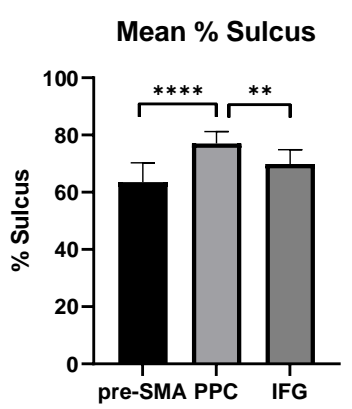

Fig. 6. Comparison of sulci percentage between VOIs. PPC showed higher percentage of sulci than pre-SMA and IFG. $* * * * \mathrm{p}<0.0001$, $\mathrm{p}<0.001 ; * *, \mathrm{p}<0.01$.

\section{DISCUSSION}

We used finite-element calculations in highly realistic head models of 12 subjects over three non-motor regions of interest: the pre-SMA, IFG and PPC. The overall results showed that, due to differences in anatomical properties between the subjects, there was not one specific coil orientation that induced the largest spatial focality within our selected VOIs. In their study, Janssen et al. [10] found better coil orientations for their studied regions, including only two of the three VOIs that we studied, using one head model for the TMS simulations without accounting for interindividual variabilities. We found that differences in the anatomical distribution of sulci and gyri 
within the VOIs is the main reason for the interindividual differences in the optimal coil orientation (Figs. 4 and 7). In fact, when analyzing the overall percentage of sulci within a VOI versus the spatial focality achieved in the same VOI, we found a positive association, increasing the spatial focality of the analyzed VOI in subjects who presented a larger percentage of sulci. In contrast, we found that spatial focality decreased when the gyri percentage increased, which is likely related to
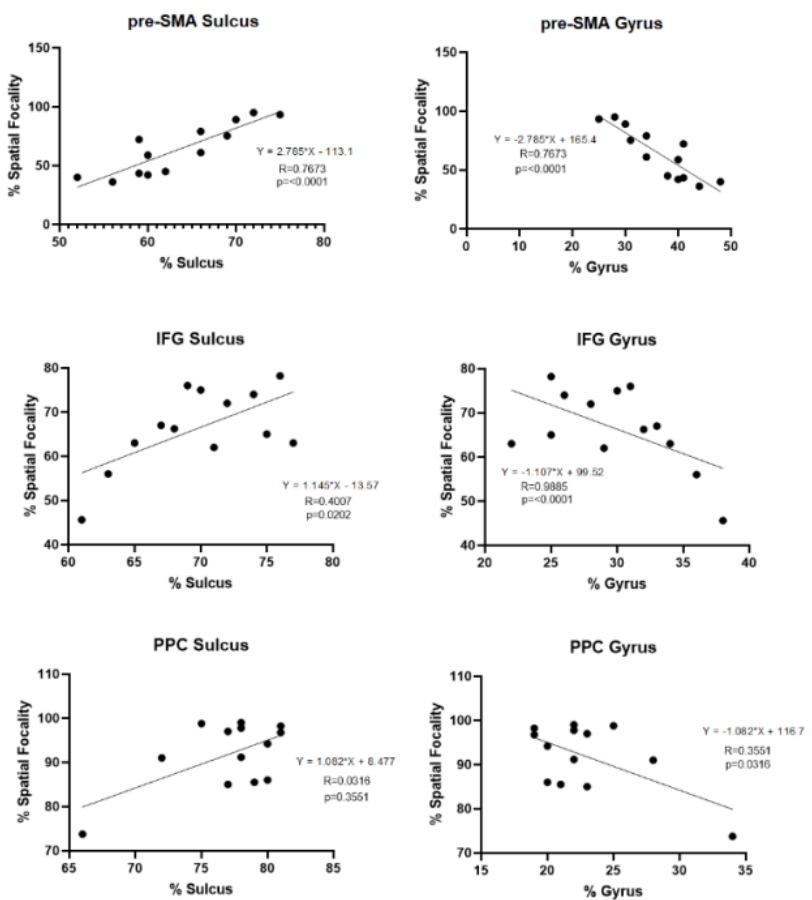

Fig. 7. Relationship between spatial focality with sulci and gyri percentages. We performed a linear regression between percentage of the overall spatial focality and percentage of sulci and gyri within our selected VOI (pre-SMA, IFG, PPC). Each dot represents the average of spatial focality for all coil directions in one particular subject. A clear relationship is shown for the three VOIs, except for the percentage of sulci in PPC $(\mathrm{P}=0.3551)$.

an increase in the perpendicular components of the current density when more sulci or walls are present in the VOI. Thus, VOIs with more and deeper walls increase the perpendicular component of the current density, which in turn generates larger spatial focality over that region. An interesting finding of this study is the 1 spatial focality in all VOIs of the PPC compared with the spatial focality achieved in the pre-SMA and IFG. When analyzing the overall percentage of sulci for each region, we found that this percentage was significantly larger in the PPC than in the pre-SMA and IFG, which is likely since the PPC presents more stable landmarks across subjects, given that the PPC includes the intraparietal sulcus [61, 62]. Thus, we showed that the PPC contains a larger percentage of sulci on average, which in turn increases the spatial focality.

Another novel approach in this study was the use of our selected VOIs based on the atlases in the MNI 152 space, which were subsequently transformed back into the native space for each subject, making the VOI selection process more accurate than when employing a fixed-radius sphere, as used in previous studies focusing on spatial focality. As the C3-model and other studies have stated [3,10,11,13,14, 17, 18, 63], cortical stimulation decreases with depth, which is due to the decrease
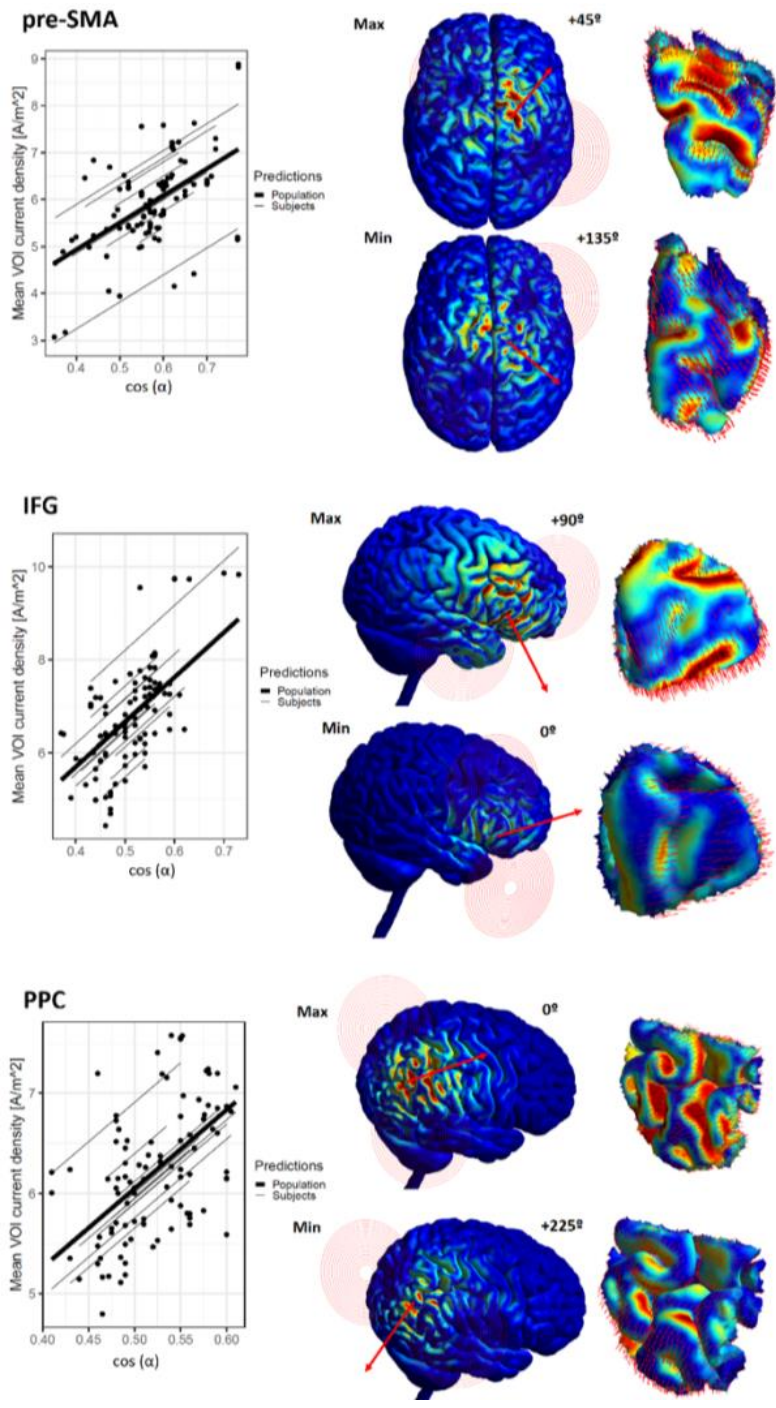

Fig. 8. Linear association between the current density and alignment of normal vector sulci with normal component of current density. Images on the left represent how the current density increases when the normal component is aligned with the normal vector of sulci. Linear mixed-effect models that includes information for the 12 head models and eight coil orientation revealed that alignment of normal vector sulci with normal component could predict current density, in the three VOIs (all p<0.0001). X-axis is the cosine of the angle $\alpha$ between the predominant orientation of the VOI sulci walls and the orientation of the coil's handle. It ranges from 0 to 1 , where a larger value means more alignment between these orientations. Images on the right show examples of maximum intensities within the VOI when normal vectors of sulci and normal components of TMS more aligned, and minimum intensities are when these components are less aligned.

in power of the current density. Therefore, only electric fields above a certain threshold of approximately $50 \mathrm{~V} / \mathrm{m}$ can generate cortical changes with sufficiently high intensity to induce a neural response, which translates into a larger effect and efficiency of the technique. In their study, Jannsen et al. analyzed coil orientations without selecting a threshold for the current density or a power percentile (being equivalent to $0 \%$ percentile in our study), considering small current densities that might be insufficient for inducing a neural response. In agreement with the authors' results and the C3-model [9], we found that the perpendicular component of the current density played an important role in generating the maximum current 
densities when selecting a certain coil orientation. Our analysis showed that when the normal vector of the sulci matches the predominant current density direction induced by the TMS (which, in the tangential figure-8 coil, is the same direction as the coil's handle), the normal-to-cortex intensity reached maximum in that region. This finding suggests an "ad-hoc" and practical orientation that is very close to optimal for increasing the intensity of the TMS stimulation without the necessity of performing TMS simulations. In other words, to achieve the largest intensity of the normal component of the current density, the handle of a figure-eight coil should be oriented perpendicularly to the orientation of the targeted gyrus. The predominant current density direction will thereby match the predominant normal vector of the sulci for a specific VOI, and the normal component of the current density penetrating the brain will be at maximum. Therefore, by knowing the direction of the gyrus to stimulate, we can predict an approximate coil position that will likely deliver the largest average intensity in the target or VOI. However, this estimate is not as accurate as the entire TMS simulation model, which is far superior (if available) in determining the best coil orientation for specific individuals due to the complex anatomical topography of the brain. Moreover, optimal coil orientations can be obtained using certain optimization approaches [64].

\section{CONCLUSION}

We studied the spatial focality and normal-to-cortex current density intensities for three non-motor TMS targets (the preSMA, IFG and PPC) in 12 detailed individualized MRI-based head models. We found no coil orientation that maximized the spatial focality across all subjects, but we did find that maximum spatial focality is obtained in the VOIs with the largest proportion of sulci. Moreover, the PPC was the target with the largest spatial focality among the three studied VOIs and was the region with the largest percentage of sulci. The largest focality is sought in repetitive TMS to reduce the side effects due to unwanted outside-VOI stimulation. The maximum averaged directional current density was obtained for coil orientations where the handle of the coil was more perpendicular to the predominant sulci orientations within the VOI.

Future experimental studies are needed to test the predictions of our approach. One possibility would be to apply dual-coil TMS to assess effective connectivity or plasticity, at different coil locations and orientations or in combination with electroencephalogram or functional MRI and measure whether different functional networks are activated. Alternatively, studies focused on behavioral and clinical outcomes can also be conducted to test the predictions of the integrated framework.

\section{REFERENCES}

[1] R. R. Birss, "Electromagnetic induction," Contemp. Phys., 1961, doi: 10.1080/00107516108202659.

[2] M. Kobayashi and A. Pascual-Leone, "Transcranial magnetic stimulation in neurology," Lancet Neurology. 2003, doi: 10.1016/S1474-4422(03)00321-1.

[3] Z. De Deng, S. H. Lisanby, and A. V. Peterchev, "Electric field depth-focality tradeoff in transcranial magnetic stimulation: Simulation comparison of 50 coil designs," Brain Stimul., 2013, doi: 10.1016/j.brs.2012.02.005.

[4] F. Fregni, D. K. Simon, A. Wu, and A. Pascual-Leone, "Non- invasive brain stimulation for Parkinson's disease: A systematic review and meta-analysis of the literature," Journal of Neurology, Neurosurgery and Psychiatry. 2005, doi:

10.1136/jnnp.2005.069849.

[5] S. Rossi et al., "Safety, ethical considerations, and application guidelines for the use of transcranial magnetic stimulation in clinical practice and research," Clinical Neurophysiology. 2009, doi: 10.1016/j.clinph.2009.08.016.

[6] P. M. Rossini and S. Rossi, "Transcranial magnetic stimulation: Diagnostic, therapeutic, and research potential," Neurology. 2007, doi: 10.1212/01.wnl.0000250268.13789.b2.

[7] M. D. Fox, H. Liu, and A. Pascual-Leone, "Identification of reproducible individualized targets for treatment of depression with TMS based on intrinsic connectivity," Neuroimage, 2013, doi: 10.1016/j.neuroimage.2012.10.082.

[8] M. S. George, "Transcranial magnetic stimulation for the treatment of depression," Expert Review of Neurotherapeutics. 2010, doi: 10.1586/ern.10.95.

[9] P. T. Fox et al., "Column-Based Model of Electric Field Excitation of Cerebral Cortex," Hum. Brain Mapp., 2004, doi: 10.1002/hbm.20006.

[10] A. M. Janssen, T. F. Oostendorp, and D. F. Stegeman, "The coil orientation dependency of the electric field induced by TMS for M1 and other brain areas," J. Neuroeng. Rehabil., 2015, doi: 10.1186/s12984-015-0036-2.

[11] I. Laakso, A. Hirata, and Y. Ugawa, "Effects of coil orientation on the electric field induced by TMS over the hand motor area," Phys. Med. Biol., 2014, doi: 10.1088/0031-9155/59/1/203.

[12] D. Antonenko, U. Grittner, G. Saturnino, T. Nierhaus, A. Thielscher, and A. Flöel, "Inter-individual and age-dependent variability in simulated electric fields induced by conventional transcranial electrical stimulation," Neuroimage, 2021, doi: 10.1016/j.neuroimage.2020.117413.

[13] I. Laakso, T. Murakami, A. Hirata, and Y. Ugawa, "Where and what TMS activates: Experiments and modeling," Brain Stimul., 2018, doi: 10.1016/j.brs.2017.09.011.

[14] S. Minjoli et al., "The impact of large structural brain changes in chronic stroke patients on the electric field caused by transcranial brain stimulation," NeuroImage Clin., 2017, doi: 10.1016/j.nicl.2017.04.014.

[15] M. Mikkonen, I. Laakso, M. Sumiya, S. Koyama, A. Hirata, and S. Tanaka, "TMS motor thresholds correlate with TDCS electric field strengths in hand motor area," Front. Neurosci., 2018, doi: 10.3389/fnins.2018.00426.

[16] A. Rotem et al., "Solving the orientation specific constraints in transcranial magnetic stimulation by rotating fields," PLoS One, 2014, doi: 10.1371/journal.pone.0086794.

[17] A. Bungert, A. Antunes, S. Espenhahn, and A. Thielscher, "Where does TMS Stimulate the Motor Cortex? Combining

Electrophysiological Measurements and Realistic Field Estimates to Reveal the Affected Cortex Position," Cereb. Cortex, 2017, doi: 10.1093/cercor/bhw292.

[18] T. D. Krieg, F. S. Salinas, S. Narayana, P. T. Fox, and D. J. Mogul, "Computational and experimental analysis of TMS-induced electric field vectors critical to neuronal activation," J. Neural Eng., 2015, doi: 10.1088/1741-2560/12/4/046014.

[19] A. Opitz, M. Windhoff, R. M. Heidemann, R. Turner, and A. Thielscher, "How the brain tissue shapes the electric field induced by transcranial magnetic stimulation," Neuroimage, 2011, doi: 10.1016/j.neuroimage.2011.06.069.

[20] T. A. Wagner, M. Zahn, A. J. Grodzinsky, and A. Pascual-Leone, "Three-dimensional head model simulation of transcranial magnetic stimulation," IEEE Trans. Biomed. Eng., 2004, doi: 10.1109/TBME.2004.827925.

[21] M. Windhoff, A. Opitz, and A. Thielscher, "Electric field calculations in brain stimulation based on finite elements: An optimized processing pipeline for the generation and usage of accurate individual head models," Hum. Brain Mapp., 2013, doi: $10.1002 / \mathrm{hbm} .21479$.

[22] A. T. Barker, R. Jalinous, and I. L. Freeston, "NON-INVASIVE MAGNETIC STIMULATION OF HUMAN MOTOR CORTEX," The Lancet. 1985, doi: 10.1016/S0140-6736(85)92413-4.

[23] J. P. Brasil-Neto, L. G. Cohen, M. Panizza, J. Nilsson, B. J. Roth, and M. Hallett, "Optimal focal transcranial magnetic activation of the human motor cortex: Effects of coil orientation, shape of the 
induced current pulse, and stimulus intensity," J. Clin. Neurophysiol., 1992, doi: 10.1097/00004691-199201000-00014.

[24] N. Eichert, K. E. Watkins, R. B. Mars, and M. Petrides,

"Morphological and functional variability in central and subcentral motor cortex of the human brain," Brain Struct. Funct., 2020, doi: 10.1101/2020.03.17.995035.

[25] F. Syeda, H. Magsood, E. G. Lee, A. A. El-Gendy, D. C. Jiles, and R. L. Hadimani, "Effect of anatomical variability in brain on transcranial magnetic stimulation treatment," AIP Adv., 2017, doi: 10.1063/1.4974981.

[26] A. Opitz, M. D. Fox, R. C. Craddock, S. Colcombe, and M. P. Milham, "An integrated framework for targeting functional networks via transcranial magnetic stimulation," Neuroimage, 2016, doi: 10.1016/j.neuroimage.2015.11.040.

[27] O. Hiwaki and T. Inoue, "A method for estimation of stimulated brain sites based on columnar structure of cerebral cortex in transcranial magnetic stimulation," J. Appl. Phys., 2009, doi: 10.1063/1.3068631.

[28] A. Pisoni, A. Vergallito, G. Mattavelli, E. Varoli, and M. Fecchio, "TMS orientation and pulse waveform manipulation activates different neural populations: direct evidence from TMS-EEG," bioRxiv, 2018, doi: 10.1101/308981.

[29] D. Ledergerber and M. E. Larkum, "Properties of layer 6 pyramidal neuron apical dendrites," J. Neurosci., vol. 30, no. 39, pp. 1303113044, 2010, doi: 10.1523/JNEUROSCI.2254-10.2010.

[30] M. M. Sorkhabi, K. Wendt, and T. Denison, "Temporally Interfering TMS: Focal and Dynamic Stimulation Location," in Proceedings of the Annual International Conference of the IEEE Engineering in Medicine and Biology Society, EMBS, 2020, doi: 10.1109/EMBC44109.2020.9176249.

[31] D. H. Benninger et al., "Intermittent theta-burst transcranial magnetic stimulation for treatment of Parkinson disease," Neurology, 2011, doi: 10.1212/WNL.0b013e31820ce6bb

[32] M. Hamada et al., "High-frequency rTMS over the supplementary motor area for treatment of Parkinson's disease," Mov. Disord., 2008, doi: $10.1002 / \mathrm{mds} .22168$.

[33] J. V. Jacobs, J. S. Lou, J. A. Kraakevik, and F. B. Horak, "The supplementary motor area contributes to the timing of the anticipatory postural adjustment during step initiation in participants with and without Parkinson's disease," Neuroscience, 2009, doi: 10.1016/j.neuroscience.2009.08.002.

[34] B. Langguth et al., "Modulating cerebello-thalamocortical pathways by neuronavigated cerebellar repetitive transcranial stimulation (rTMS)," Neurophysiol. Clin., 2008, doi:

10.1016/j.neucli.2008.08.003.

[35] M. Mulckhuyse, T. A. Kelley, J. Theeuwes, V. Walsh, and N. Lavie, "Enhanced visual perception with occipital transcranial magnetic stimulation," Eur. J. Neurosci., 2011, doi: 10.1111/j.14609568.2011.07814.x.

[36] G. Pobric and A. F. d. C. Hamilton, "Action understanding requires the left inferior frontal cortex," Curr. Biol., 2006, doi: 10.1016/j.cub.2006.01.033.

[37] D. Konakanchi, A. L. de Jongh Curry, R. S. Waters, and S. Narayana, "Focality of the induced e-field is a contributing factor in the choice of tms parameters: Evidence from a 3d computational model of the human brain," Brain Sci., vol. 10, no. 12, pp. 1-17, 2020, doi: 10.3390/brainsci10121010.

[38] W. H. Lee, S. H. Lisanby, A. F. Laine, and A. V. Peterchev, "Electric Field Model of Transcranial Electric Stimulation in Nonhuman Primates: Correspondence to Individual Motor Threshold," IEEE Trans. Biomed. Eng., 2015, doi:

10.1109/TBME.2015.2425406.

[39] N. Arai, M. K. Lu, Y. Ugawa, and U. Ziemann, "Effective connectivity between human supplementary motor area and primary motor cortex: A paired-coil TMS study," Exp. Brain Res., 2012, doi: 10.1007/s00221-012-3117-5.

[40] A. R. E. Potgieser, B. M. de Jong, M. Wagemakers, E. W. Hoving, and R. J. M. Groen, "Insights from the supplementary motor area syndrome in balancing movement initiation and inhibition," Front. Hum. Neurosci., 2014, doi: 10.3389/fnhum.2014.00960.

[41] V. MacDonald and G. M. Halliday, "Selective loss of pyramidal neurons in the pre-supplementary motor cortex in Parkinson's disease," Movement Disorders. 2002, doi: 10.1002/mds.10258.

[42] A. R. Aron, P. C. Fletcher, E. T. Bullmore, B. J. Sahakian, and T. W. Robbins, "Stop-signal inhibition disrupted by damage to right inferior frontal gyrus in humans," Nat. Neurosci., 2003, doi: 10.1038/nn1003.

[43] A. Hampshire, S. R. Chamberlain, M. M. Monti, J. Duncan, and A. M. Owen, "The role of the right inferior frontal gyrus: inhibition and attentional control," Neuroimage, 2010, doi: 10.1016/j.neuroimage.2009.12.109.

[44] P. Porcacchia et al., "Parieto-motor cortical dysfunction in primary cervical dystonia," Brain Stimul., 2014, doi: 10.1016/j.brs.2014.06.007.

[45] G. B. Saturnino, O. Puonti, J. D. Nielsen, D. Antonenko, K. H. Madsen, and A. Thielscher, "SimNIBS 2.1: A Comprehensive Pipeline for Individualized Electric Field Modelling for Transcranial Brain Stimulation," in Brain and Human Body Modeling, 2019.

[46] J. Gomez-Feria et al., "3D Printing of Diffuse Low-Grade Gliomas Involving Eloquent Cortical Areas and Subcortical Functional Pathways: Technical Note," World Neurosurg., 2021, doi: 10.1016/j.wneu.2020.12.082.

[47] J. Sallet et al., "The organization of dorsal frontal cortex in humans and macaques," J. Neurosci., 2013, doi:

10.1523/JNEUROSCI.5108-12.2013.

[48] R. S. Desikan et al., "An automated labeling system for subdividing the human cerebral cortex on MRI scans into gyral based regions of interest," Neuroimage, 2006, doi:

10.1016/j.neuroimage.2006.01.021.

[49] J. Talairach and P. Tournoux, Co-planar stereotaxic atlas of the human brain: Threedimensional proportional system: An approach to cerebral imaging. 1988.

[50] J. L. Lancaster et al., "Bias between MNI and talairach coordinates analyzed using the ICBM-152 brain template," Hum. Brain Mapp., 2007, doi: $10.1002 / \mathrm{hbm} .20345$.

[51] M. W. Woolrich et al., "Bayesian analysis of neuroimaging data in FSL.," Neuroimage, 2009, doi: 10.1016/j.neuroimage.2008.10.055.

[52] S. M. Smith et al., "Advances in functional and structural MR image analysis and implementation as FSL," in NeuroImage, 2004, doi: 10.1016/j.neuroimage.2004.07.051.

[53] M. Jenkinson, C. F. Beckmann, T. E. J. Behrens, M. W. Woolrich, and S. M. Smith, "Review FSL," Neuroimage, 2012, doi: 10.1016/j.neuroimage.2011.09.015.

[54] W. Wang and S. R. Eisenberg, "A Three-Dimensional Finite Element Method for Computing Magnetically Induced Currents in Tissues," IEEE Trans. Magn., 1994, doi: 10.1109/20.334289.

[55] M. Fernández-Corazza, S. Turovets, and C. H. Muravchik, "Unification of optimal targeting methods in transcranial electrical stimulation," Neuroimage, 2020, doi

10.1016/j.neuroimage.2019.116403.

[56] M. Fernández-Corazza, L. Beltrachini, N. Von Ellenrieder, and C. H. Muravchik, "Analysis of parametric estimation of head tissue conductivities using Electrical Impedance Tomography," Biomed. Signal Process. Control, 2013, doi: 10.1016/j.bspc.2013.08.003.

[57] F. S. Salinas, J. L. Lancaster, and P. T. Fox, "Detailed 3D models of the induced electric field of transcranial magnetic stimulation coils," Phys. Med. Biol., 2007, doi: 10.1088/0031-9155/52/10/016.

[58] A. Thielscher and T. Kammer, "Electric field properties of two commercial figure- 8 coils in TMS: Calculation of focality and efficiency," Clin. Neurophysiol., 2004, doi:

10.1016/j.clinph.2004.02.019.

[59] Y. H. Kwon and H. Bang, "The Finite Element Method Using MATLAB, Second Edition," CRC Press. 2000.

[60] T. Preston, "Finite Elements for Electrical Engineers," Comput. Eng. J., 1984, doi: 10.1049/cae.1984.0027.

[61] J. R. Whitlock, "Posterior parietal cortex," Current Biology. 2017, doi: 10.1016/j.cub.2017.06.007.

[62] J. D. Swisher, M. A. Halko, L. B. Merabet, S. A. McMains, and D. C. Somers, "Visual topography of human intraparietal sulcus," $J$. Neurosci., vol. 27, no. 20, pp. 5326-5337, 2007, doi: 10.1523/JNEUROSCI.0991-07.2007.

[63] L. Richter, G. Neumann, S. Oung, A. Schweikard, and P. Trillenberg, "Optimal Coil Orientation for Transcranial Magnetic Stimulation," PLoS One, 2013, doi: 10.1371/journal.pone.0060358.

[64] L. J. Gomez, M. Dannhauer, and A. V. Peterchev, "Fast computational optimization of TMS coil placement for individualized electric field targeting," Neuroimage, 2021, doi: 10.1016/j.neuroimage.2020.117696. 